\title{
Sonification of lattice data: The spectrum of the Dirac operator across the deconfinement transition
}

\author{
Alberto de Campo \\ Institute of Electronic Music and Acoustics, University for Music and Dramatic Arts, Graz, \\ Austria \\ E-mail: decampo@iem.at
}

\section{Natascha Hörmann}

Atominstitut, Vienna University of Technology, Austria

E-mail: e9026090estudent.tuwien.ac.at

\section{Harald Markum ${ }^{* \dagger}$}

Atominstitut, Vienna University of Technology, Austria

E-mail: markumetuwien.ac.at

\section{Willibald Plessas}

Theoretical Physics, Institute of Physics, University of Graz, Austria

E-mail: willibald.plessas@uni-graz.at

\section{Bianka Sengl}

Theoretical Physics, Institute of Physics, University of Graz, Austria

E-mail: bianka.sengleuni-graz.at

Sonification is the use of non-speech audio to extract information from data. It represents the sound analogue to graphical visualization. The method is applied in several disciplines from economy to medicine to physics. Sonification might also help in the analyzation process of lattice data. It could assist, together with graphical display, to examine the behavior of lattice observables as a function of parameters like gauge coupling, quark mass, etc. It might further be used to point out unique characteristics of single gauge field configurations out of many. In order to demonstrate the methodology for quantum chromodynamics (QCD) we analyze the eigenvalues of the Dirac operator from the confinement to the deconfinement phase. We are adapting a program package for audio browsing of baryon spectra from quark models developed at the University of Graz within the interdisciplinary research project 'SonEnvir'.

XXIIIrd International Symposium on Lattice Field Theory

25-30 July 2005

Trinity College, Dublin, Ireland

\footnotetext{
*Speaker.

†The project 'SonEnvir' (http://sonenvir.at/) is fi nanced by the Future Fund of the Province of Styria, Austria.
} 


\section{Visualization and Sonification}

In many areas of science and economy a vast amount of relevant data is stored and made available for evaluation. Techniques to locate the numbers of interest like data warehouse and data mining have been developed together with program packages for visualization in order to extract the hidden information. The question arose if methods of auditory display could help to get insight into the structures behind the data. This discipline is called sonifi cation and a defi nition can be found in the wikipedia: Sonifi cation is the use of non-speech audio to convey information or perceptualize data (http://en.wikipedia.org/wiki/Sonifi cation). The International Community for Auditory Display (ICAD) (http://www.icad.org/) was established in 1992 and has been organizing international conferences since then; this year's conference took place in Limerick, Ireland, a few days before the lattice conference in Dublin. At the request of the NSF in 1997 the ICAD provided a Sonifi cation Repor.

\section{Sonification Package}

Sonifi cation needs a realization on software and hardware platforms. We will describe the program package SuperCollider developed by James McCartney from Austin, TX. It originated as proprietary software and was released in 2002 under the free software GPL license. The name SuperCollider is said to have its origin from the Superconducting Super Collider (SSC) in Waxahachie, TX, which was planned and begun to be constructed but was then abandoned and never fi nished.

The SuperCollider environment consists of two applications, a client sclang (that is the language) and a server scsynth (that is the audio) which communicate using Open Sound Control. The SuperCollider language (sclang) is an interpreter language and combines the object oriented structure of Smalltalk and features from functional programming languages with a $\mathrm{C}$ programming language family syntax. SuperCollider runs on the platforms Mac OS X, Linux, and Windows. Relying on Linux, the program code can interactively be edited and executed using, e.g., the emacs editor. The synthesizer (scsynth) can make use of the ALSA sound system. We mention that beside this professional solution there is a simple sonifi cation tool implemented in newer versions of Mathematica, and MATLAB supports treating data vectors as if they were audio signals and playing them.

\section{Application to Lattice QCD}

In order to study the possible use of sonifi cation in lattice fi eld theory we took as an example the eigenvalue spectrum of the Dirac operator from existing data [i]. We generated gauge fi eld confi gurations using the standard Wilson plaquette action for SU(3) and constructed the matrix of the Dirac operator using the Kogut-Susskind prescription. The Dirac matrix is anti-hermitian so that all eigenvalues are imaginary and occur in pairs with opposite sign. We worked on a $6^{3} \times 4$ lattice with various values of $\beta$ and typically produced 10 independent confi gurations for each $\beta$.

Figure 11 shows a graphical presentation of the 15 lowest eigenvalues from $\beta=5.0$ to $\beta=6.0$. The phase transition to deconfi nement occurs around $\beta=5.7$ where all quasi-zero modes vanish. 
Dirac eigenvalues

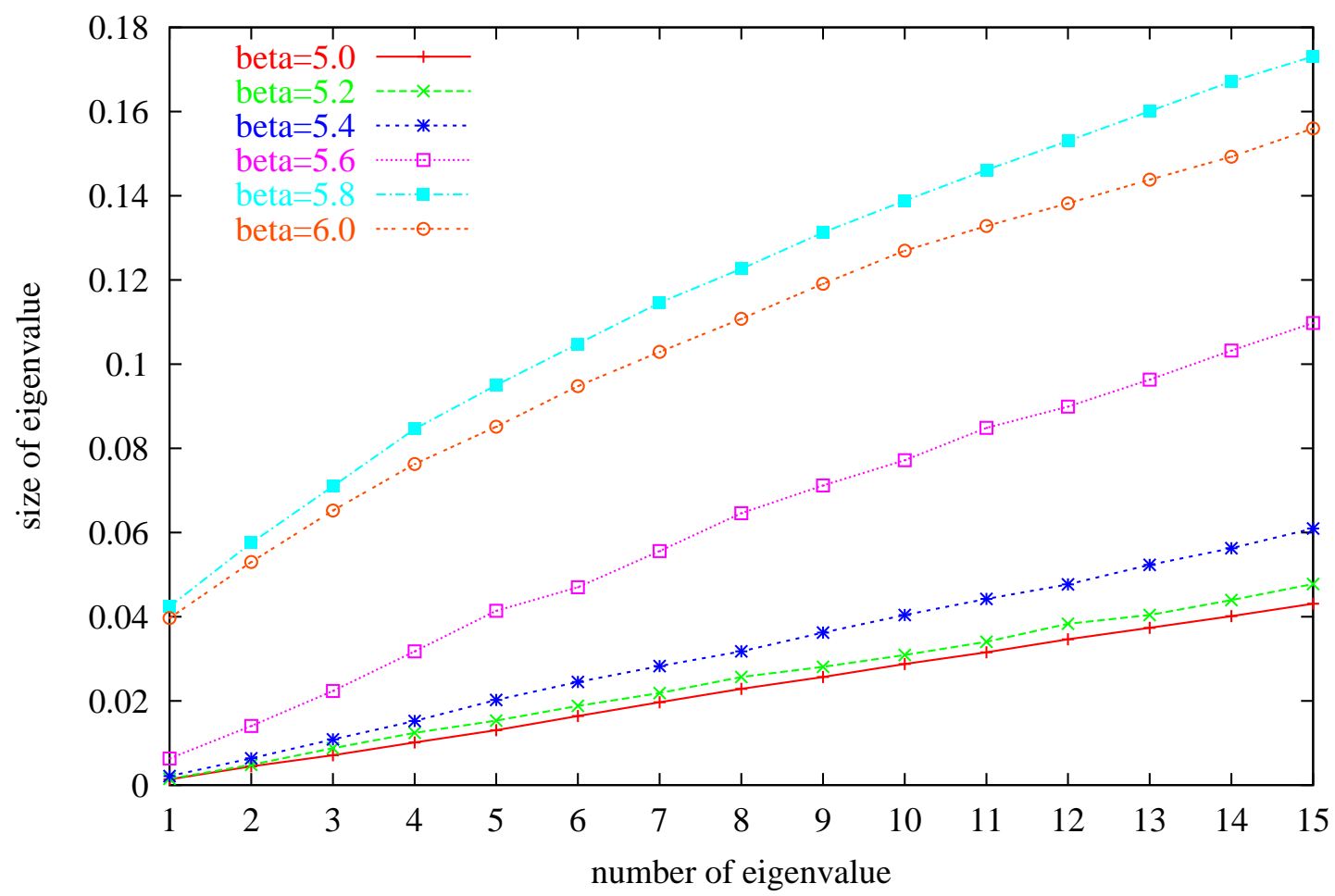

Figure 1: Configuration averaged values of the 15 lowest levels of the eigenvalue spectrum of the Dirac operator for 6 different couplings $\beta$.

For the auditory presentation we multiplied the raw data by a factor of 10000 and added the standard pitch of $440 \mathrm{~Hz}$. The outcome is shown in Fig. 2 for the spectra from the confi nement to the deconfi nement phase.

In the sonifi cation process we modifi ed a sclang code from another sonifi cation project treating baryon spectra obtained from different constituent quark models [2]. In Fig. 3 we present screenshots of the 15 lowest eigenvalues for $\beta=5.0$ and $\beta=6.0$. Listening to the sound fi les one can hear that the so-called melody is rather similar for the $\beta$-values in the confi nemen phase with a slight increase towards higher coupling. The quasi-zero mode of the lowest eigenvalue stays around 440 Hz. After the transition to the deconfinemen region the melody changes clearly to higher tones. The lowest eigenvalue starts above $800 \mathrm{~Hz}$. This means that one can hear the restoration of chiral symmetry when increasing the coupling to the quark-gluon plasma-phase. These sample results are stored on the SonEnvin server and can be accessed there.

\section{What One Has Learned}

This contribution reports fi rst attempts of applying auditory display to data from lattice QCD. The aim has been to fi nd possible advantages in the data analysis through sonifi cation. Using the example of the eigenvalue spectrum of the Dirac operator some primitive sound files have been generated that allow to hear evident features, which have been familiar already from other kinds 
Dirac eigenvalues

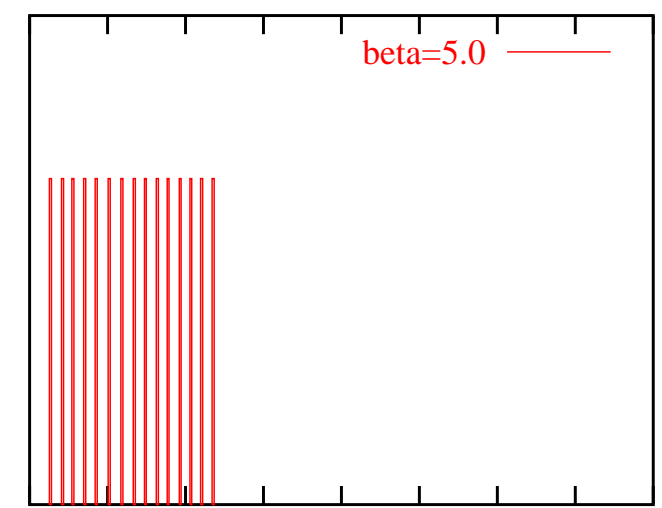

400600800100012001400160018002000 frequency

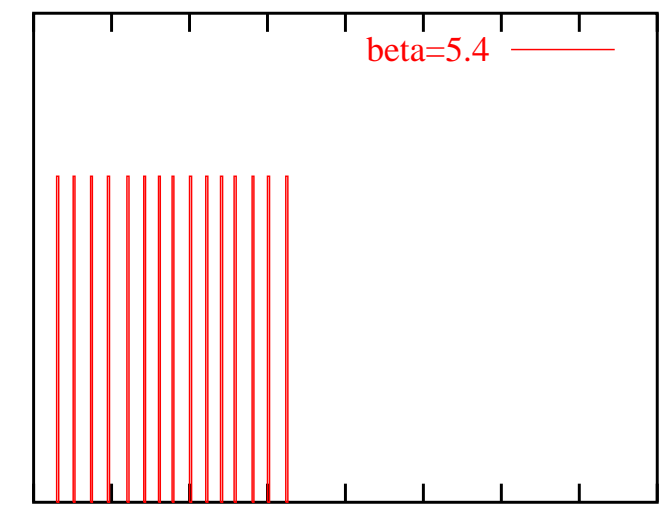

$400 \quad 600800100012001400160018002000$ frequency

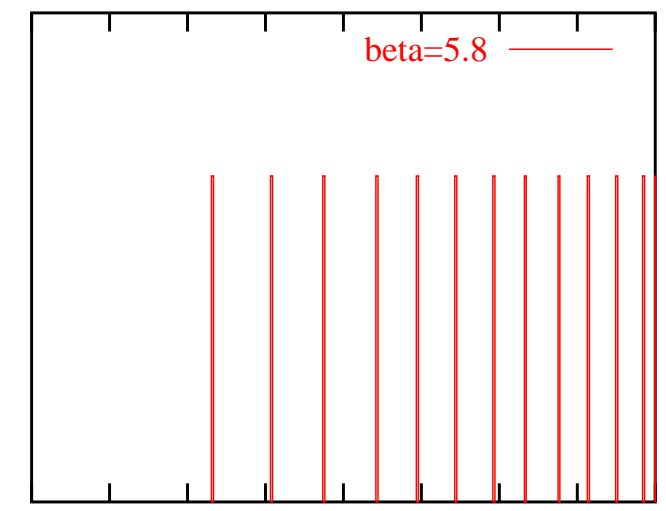

$400 \quad 600800100012001400160018002000$ frequency beta $=[5.0,5.2,5.4,5.6,5.8,6.0]$
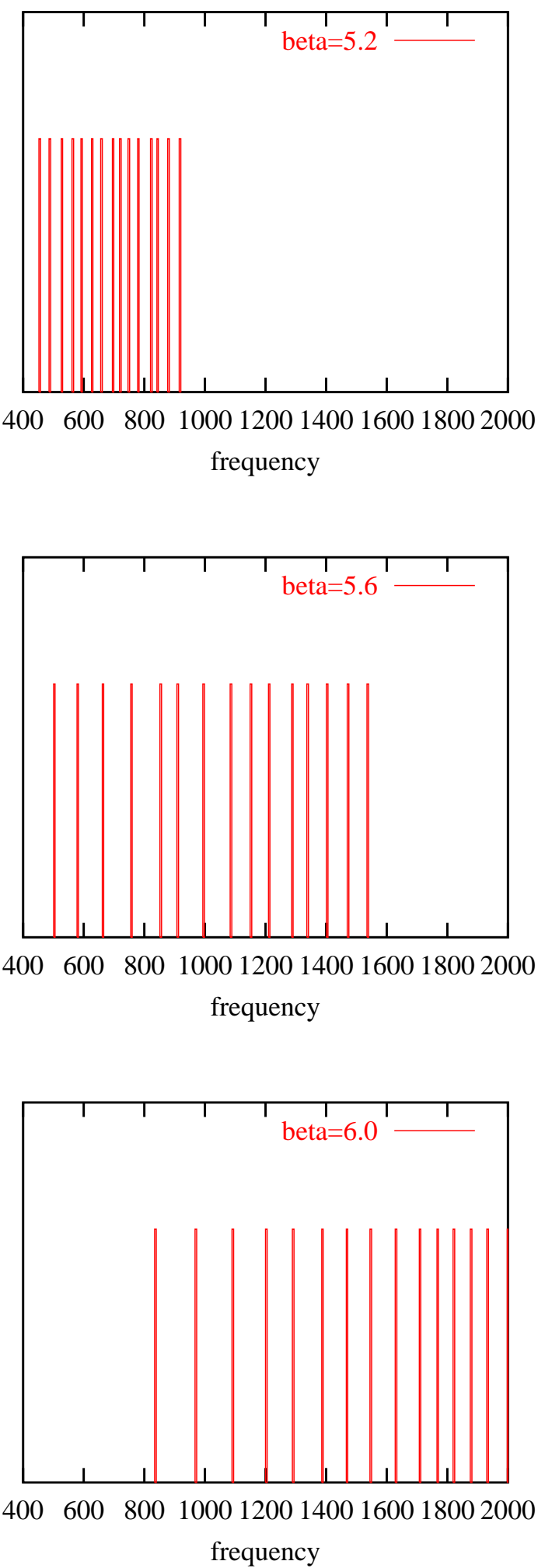

Figure 2: Eigenvalue spectra transformed into audible region for $\beta$-values across the phase transition from quark confinement to the quark-gluon plasma. 

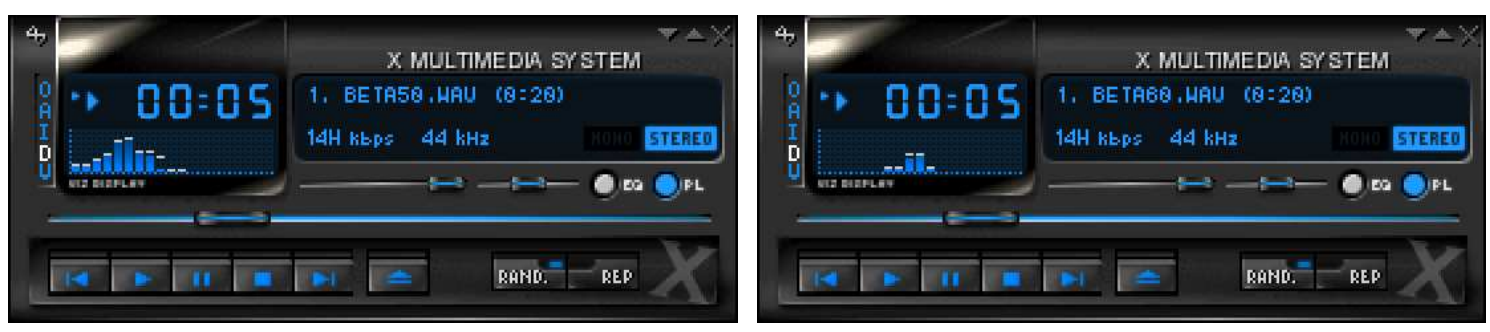

Figure 3: Screenshots of the low-lying part of the spectrum of the Dirac operator in the confinement and deconfinement regions.

of data analysis, in particular, from graphical visualization. In this regard, sonifi cation as applied here can be seen as an additional tool of data representation. Of course, one should fi nd more refi ned means of auditory display in order to make further qualities apparent in some given data sets. Sonifi cation offers the chance to detect structures in the data sets that have been hidden to the methods applied so far. Data analysis through sonifi cation might especially be useful for displaying results depending on multiple parameters and/or belonging to higher space-time dimensions. In the context of lattice QCD one could think, e.g., of the investigation of the topological content of certain gauge fi eld confi gurations.

\section{Acknowledgment}

We acknowledge the assistance of Rainer Pullirsch in producing the eigenvalue spectra of the Dirac operator. The speaker thanks Philippe de Forcrand for making him aware of the sonifi cation tool in Mathematica.

\section{References}

[1] R. Pullirsch, K. Rabitsch, T. Wettig, H. Markum, Evidence for quantum chaos in the plasma phase of QCD, Phys. Lett. B427 (1998) 119 [hep-ph/9803285].

[2] A. de Campo, C. Frauenberger, R. Höldrich, T. Melde, W. Plessas, B. Sengl, Sonification of quantum spectra, in Proceedings of ICAD 05 - Eleventh Meeting of the International Conference on Auditory Display, Limerick, Ireland, July 6-9, 2005

[http://www.idc.ul.ie/icad2005/downloads/f67.pdf]. 